\title{
Knowledge, attitude and practice of adverse drug reaction reporting among healthcare professionals of a tertiary care hospital in Gujarat
}

\author{
Nirav N. Patel, Devanshu J. Patel*, Haresh A. Desai
}

Department of Pharmacology, Parul Institute of Medical Sciences and Research Limda, Vadodara, Gujarat India

Received: 05 September 2019 Revised: 18 September 2019 Accepted: 19 September 2019

*Correspondence to: Dr. Devanshu J. Patel, Email: president@parul university.ac.in

Copyright: (C) the author(s), publisher and licensee Medip Academy. This is an openaccess article distributed under the terms of the Creative Commons Attribution NonCommercial License, which permits unrestricted noncommercial use, distribution, and reproduction in any medium, provided the original work is properly cited.

\begin{abstract}
Background: The objective of the present study was to assess knowledge, attitude and practice of adverse drug reaction reporting among healthcare professionals of Parul Sevashram Hospital, Parul University, Vadodara.

Methods: The study was prospective, observational and questionnaire-based involving medical doctors, nurses and pharmacists of Parul Sevashram Hospital. A total number of 210 structured questionnaires were distributed among health care professionals (100 clinicians from various specialities, 100 nurses and 10 pharmacists). Written informed consent forms were obtained from participants as their consent to participate in the study.

Results: All questionnaires were filled and returned producing overall response rate of $100 \%$. $80 \%$ of the respondents were aware of the existence of Pharmacovigilance Program of India (PvPI). $90.95 \%$ and $91.90 \%$ of respondents felt ADR reporting is necessary and it would be beneficial to the patients respectively. Only $11.43 \%$ of respondents had reported ADRs. $90.95 \%$ and $85.24 \%$ of respondents suggested training is required in reporting ADR and regular information should be provided regarding ADR by PvPI respectively.

Conclusions: The health care professionals had positive knowledge and attitude towards pharmacovigilance and there is a need to create awareness for better practice of ADR reporting. It would help to improve patient care.
\end{abstract}

Keywords: Adverse drug reactions, Pharmacovigilance, Knowledge, Practice

\section{INTRODUCTION}

According to World Health Organisation, an adverse drug reaction is defined as "a response to a drug which is noxious and unintended and which occurs at doses normally used in man for prophylaxis, diagnosis or therapy of disease or for the modification of physiological function excluding failure to accomplice the intended purpose". ${ }^{1}$ Adverse drug reactions (ADRs) are one of the major drug related problems associated with pharmacotherapy. Pharmacovigilance is defined as the science and activity relating to detection, assessment, understanding and prevention of adverse effects or any other possible drug related problems. ${ }^{2}$ Spontaneous adverse drug reaction reporting is the integral part of Pharmacovigilance Programme of India (PvPI) in the post-approval phase. To increase spontaneous adverse drug reaction reporting, involvement of the healthcare professionals for reporting of ADRs is necessary. With respect to developed countries, despite of better ADR reporting culture, under-reporting is a major issue with spontaneous reporting. ${ }^{3,4}$ Under reporting delays early detection of ADRs and increase morbidity and mortality in patients. ${ }^{5}$ Major factor for under-reporting might be a lack of awareness among healthcare professionals towards the existing pharmacovigilance program. In order to improve the reporting rate, it is important to improve the knowledge, attitude and practices of the 
healthcare professionals regarding ADR reporting and Pharmacovigilance. This would help us in planning interventions amongst health care professionals. It is for this purpose we planned this to evaluate the knowledge, attitude and practice of ADR reporting by physicians, nurses and pharmacists.

\section{METHODS}

A prospective observational (non-interventional) questionnaire-based study was conducted over a period of two months from $1^{\text {st }}$ June 2019 to $31^{\text {st }}$ July 2019 among health care professionals of Parul Sevashram Hospital- a tertiary care teaching hospital, Parul University, Vadodara, Gujarat. This study was started after receiving due permission from Parul University Institutional Ethics Committee for Human Research. Written informed consent forms were obtained from participants as their consent to participate in the study. The prospective participants were provided all the information about the objective and nature of the study in the language understood by them. A total number of 210 structured questionnaires were distributed among 100 clinicians from various specialities, 10 pharmacists and 100 nurses. The pre-designed questionnaire developed from previous study to assess knowledge, attitude and practice of ADR reporting by practitioners, pharmacists and nurses. ${ }^{6,7}$ The questionnaire comprised of 20 questions related to knowledge, attitude and practice of healthcare care professionals, 6 questions related to factors responsible for non-reporting and 4 questions related to factors responsible for encouragement of ADR reporting. A time frame of one week was allotted to the participants for the collection of the anonymously filled forms. Collected data was entered and analysed using Microsoft Excel and the findings was presented in number and percentages.

\section{RESULTS}

A total of 210 questionnaires were distributed among various health care professionals of Parul Sevashram Hospital, Vadodara, Gujarat, India. All questionnaires were filled and returned producing overall response rate of $100 \%$.

Table 1: Knowledge, attitude and practice of healthcare professionals.

\begin{tabular}{|c|c|c|c|c|c|}
\hline S. no & Question & Yes & $\%$ & No & $\%$ \\
\hline 1 & All drugs available in the market are safe. & 29 & 13.81 & 181 & 86.19 \\
\hline 2 & All ADRs should be reported for all drugs. & 60 & 28.57 & 150 & 71.43 \\
\hline 3 & Do you think reporting of adverse drug reaction is necessary? & 191 & 90.95 & 19 & 9.05 \\
\hline 4 & The health care professionals are responsible for ADR reporting. & 168 & 80.00 & 42 & 20.00 \\
\hline 5 & Have you ever come across with an ADR? & 150 & 71.43 & 60 & 28.57 \\
\hline 6 & I have reported an ADR before. & 24 & 11.43 & 186 & 88.57 \\
\hline 7 & I have been trained on how to report an ADR. & 29 & 13.81 & 181 & 86.19 \\
\hline 8 & Training is needed in reporting an ADR. & 191 & 90.95 & 19 & 9.05 \\
\hline 9 & $\begin{array}{l}\text { I have seen the suspected ADR reporting form (white form) issued by } \\
\text { CDSCO. }\end{array}$ & 125 & 59.52 & 85 & 40.48 \\
\hline 10 & I am aware of the nearest AMC in my geographical location. & 30 & 14.29 & 180 & 85.71 \\
\hline 11 & I am aware of the existence of PvPI. & 168 & 80.00 & 42 & 20.00 \\
\hline 12 & ADR reporting is a professional obligation. & 173 & 82.38 & 37 & 17.62 \\
\hline 13 & ADR reporting should be made mandatory to my profession. & 189 & 90.00 & 21 & 10.00 \\
\hline 14 & $\begin{array}{l}\text { Only serious adverse event or increased frequency of an ADR of old drugs } \\
\text { needs to be reported. }\end{array}$ & 162 & 77.14 & 48 & 22.86 \\
\hline 15 & $\begin{array}{l}\text { Reporting of only one ADR makes no significant contribution to the PvPI } \\
\text { or society. }\end{array}$ & 141 & 67.14 & 69 & 32.86 \\
\hline 16 & $\begin{array}{l}\text { Have you ever come across educational session in specific about } \\
\text { pharmacovigilance or ADRs? }\end{array}$ & 142 & 67.62 & 68 & 32.38 \\
\hline 17 & ADR reporting and monitoring system would beneficial to the patient. & 193 & 91.90 & 17 & 8.10 \\
\hline 18 & Regular information regarding ADR should be provided by PvPI? & 179 & 85.24 & 31 & 14.76 \\
\hline 19 & Do you support "direct ADR reporting" by the patients? & 177 & 84.29 & 33 & 15.71 \\
\hline 20 & $\begin{array}{l}\text { Do you think pharmacovigilance should be taught in detail to healthcare } \\
\text { professionals? }\end{array}$ & 173 & 82.38 & 37 & 17.62 \\
\hline
\end{tabular}

In our study, while assessing the knowledge of the participants, we found that $125(59.52 \%)$ participants had seen the suspected adverse drug reaction reporting form (white form) issued by Central Drug Standard Control Organization (CDSCO). One hundred and sixty eight
$(80 \%)$ of the participants were aware of the existence of PvPI, however, 180 (85. 71\%) participants did not know the nearest Adverse drug reaction monitoring center (AMC). Further analysis relating to knowledge of the participants showed that majority of them agreed that all 
drugs are not safe $(181(86.19 \%))$ and ADR reporting system would beneficial to the patient (193 (91.90\%)). However, $150(71.43 \%)$ participants did not agree that all ADRs should be reported for all drugs. In the present study, $162(77.14 \%)$ and $141(67.14 \%)$ participants felt that only serious adverse events are needed to be reported and reporting of single ADR makes no significant contribution to the PvPI respectively (Table 1).

In respect of attitude of health care professionals, majority of participants agreed that reporting of adverse drug reaction is necessary $(191(90.95 \%))$ and health care professionals are responsible for the reporting (168 $(80 \%))$. One hundred and seventy three (82.38\%) participants felt that ADR reporting is a professional obligation and ADR reporting should be made mandatory to my profession (189 (90\%)) (Table 1).

In respect of practice of health care professionals, our study results revealed that a total of 150 (71.43\%) participants had come across the ADR, however, only 24 $(11.43 \%)$ had reported ADRs. It was found that only 29 (13\%) participants were trained in reporting ADR. One hundred and ninety one $(90.95 \%)$ participants suggested training is required in reporting ADR. Further, our study showed that $143(67.62 \%)$ participants had come across educational session in specific about pharmacovigilance and $179(85.24 \%)$ participants suggested that regular information should be provided regarding ADR by PvPI (Table 1).

Table 2: Factors contributed to non-reporting of ADR.

\begin{tabular}{|llllll|}
\hline S. no & Question & Yes & $\%$ & No & $\%$ \\
\hline $\mathbf{1}$ & Do not know how to report, where to report and when to report & 148 & 70.48 & 62 & 29.52 \\
\hline $\mathbf{2}$ & Extra work burden & 123 & 58.57 & 87 & 41.42 \\
\hline $\mathbf{3}$ & Concern that the report may be wrong (fear) & 87 & 41.42 & 123 & 58.57 \\
\hline $\mathbf{4}$ & Lack of time to fill the ADR form & 67 & 31.90 & 143 & 68.09 \\
\hline $\mathbf{5}$ & Failure of spoiling reputation & 45 & 21.42 & 165 & 78.57 \\
\hline $\mathbf{6}$ & Lack of confidence (for considering as failure in part of treatment) & 35 & 16.66 & 175 & 83.33 \\
\hline
\end{tabular}

Table 3: Factors contributed to encouragement of reporting ADR.

\begin{tabular}{|c|c|c|c|c|c|}
\hline S. no & Question & Yes & $\%$ & No & $\%$ \\
\hline 1 & If the ADR is serious. & 149 & 70.95 & 61 & 29.05 \\
\hline 2 & If the ADR is unusual. & 142 & 67.62 & 68 & 32.38 \\
\hline 3 & If the ADR is due to a new product. & 179 & 85.24 & 31 & 14.76 \\
\hline 4 & If the ADR is well recognized for a particular drug. & 160 & 76.19 & 50 & 23.81 \\
\hline
\end{tabular}

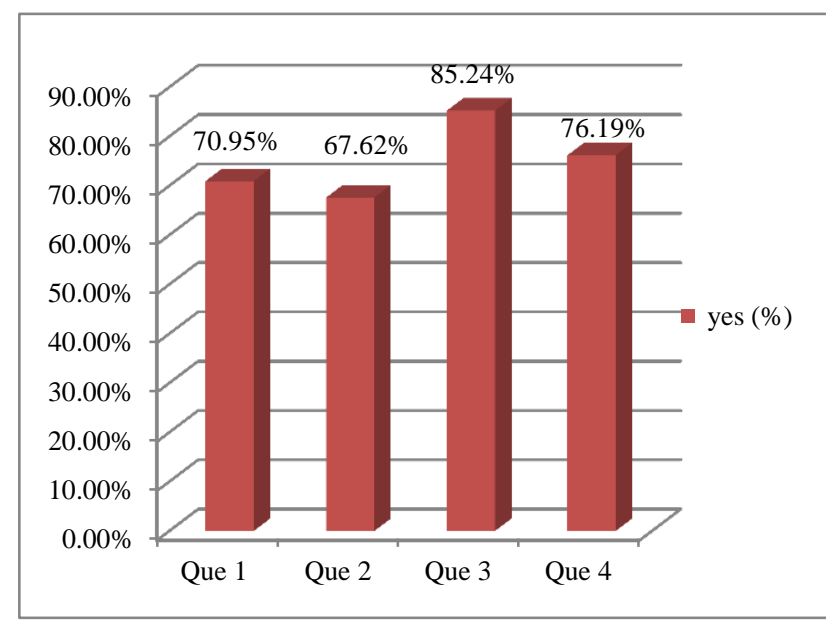

Figure 1: Factors contributed to encouragement of reporting ADR.

In our study, factors contributed to non-reporting of ADR were lack of awareness about how, where and when to report $(148(70.48 \%))$, extra work burden (123 $(58.57 \%))$, concern that the report may be wrong (fear) (87 $(41.42 \%))$, lack of time to fill ADR (67 (31.90\%)), failure of spoiling reputation $(45(21.42 \%))$ and lack of confidence (for considering as failure in part of treatment) (35 (16.66\%)) (Table 2). Whereas common factors that lead to enhancement ADR reporting were if the ADR is serious (149 (70.95\%)), unusual (142 $(67.62 \%))$, due to a new drug product $(179(85.24 \%))$ and well recognized for a particular drug (160 (76.19\%)) (Table 3 and Figure 1).

\section{DISCUSSION}

Healthcare professionals play a key role in understanding and reporting of adverse drug reactions. Adverse drug reactions are routinely encountered in hospital set up, however, they are not being reported. ${ }^{8}$ The barriers to ADR reporting can be a lack of knowledge and awareness regarding pharmacovigilance as well as attitude in the health care professionals.

In our study, it was observed that health care professionals had positive knowledge and attitudes towards pharmacovigilance which was found lower in other study done in India. ${ }^{7}$ In the present study, around $60 \%$ and $80 \%$ of healthcare professionals had seen suspected adverse drug reaction reporting form (white form) issued by $\mathrm{CDSCO}$ used to report ADR and were 
aware of the existence of Pharmacovigilance Program of India respectively. Although around $91 \%$ of the health care professionals felt that ADR reporting is necessary, $82.37 \%$ of them had considered reporting a professional obligation. These findings were consistent with the study done by Ganesan et al. ${ }^{9}$ Despite of positive knowledge and attitude of health care professionals found in our study, ADR reporting was approximately $12 \%$. In similar studies done by Palaian et al and Okezie et al, it was found that ADR reporting was $33.7 \%$ and $32 \%$ respectively. ${ }^{10,11}$

In many studies, it was observed that common factors responsible for non-reporting of ADRs included lack of awareness about pharmacovigilance (what, how and when to report), inadequate information about ADR, extra work burden, concern that report may be wrong and lack of time..$^{5}$ In our study, we found similar results, common factors found were lack of knowledge and awareness (70.48\%), extra work burden (58.57\%), concern that the report may be wrong (fear) $(41.42 \%)$ and lack of confidence (for considering as failure in part of treatment) (16.66\%). Regular education and training programme about pharmacovigilance and reporting of ADR as well as exchange of information between national pharmacovigilance centers and health care providers would help to overcome these barriers, improve ADR reporting and strengthen the activates of pharmacovigilance programme of India. However, there were few factors that contributed to encouragement of ADR reporting included if the reaction is due to a new drug product and well recognized for a particular drug. These findings were comparable to study done by Gupta et al. ${ }^{12}$

Effective implementation of pharmacovigilance activities by improving knowledge, attitude and practice of health care professionals can help us to promote safe and rational use of medicines.

\section{CONCLUSION}

Despite of favourable knowledge and attitude among healthcare professionals, there is a need to create awareness about the importance ADRs reporting scrupulously and without reticence. This practice will prove very valuable in promoting the safe and rational use of drug therapy. Many untoward adverse incidents pass unnoticed, due to the lack of awareness, extra burden, clinical acumen, aptitude and/or time. This study will help in identifying such barriers in reporting ADRs and can contribute effectively towards improving ADR reporting.

Funding: No funding sources Conflict of interest: None declared

Ethical approval: The study was approved by the Institutional Ethics Committee

\section{REFERENCES}

1. Tripathi KD. Essentials of medical pharmacology. 6th ed. New Delhi, India: Jaypee Publications; 2008.

2. WHO Policy Perspectives on Medicines. Pharmacovigilance: ensuring the safe use of medicines. Geneva: World Health Organization; 2004.

3. Lopez-Gonzalez E, Herdeiro MT, Figueiras A. Determinants of under-reporting of adverse drug reactions: a systematic review. Drug Saf. 2009;32:1931.

4. Williams D, Feely J. Underreporting of adverse drug reactions: attitudes of Irish doctors. Ir J Med Sci. 1999;168:257-61.

5. John LJ, Arifulla M, Cheriathu JJ, Sreedharan J. Reporting of adverse drug reactions: an exploratory study among nurses in a teaching hospital, Ajman, United Arab Emirates. DARU J Pharmaceut Sci. 2012;20(1):44.

6. Desai CK, Iyer PG, Panchal J, Shah S, Dikshit RK. An evaluation of knowledge, attitude, and practice of adverse drug reaction among prescriber at a tertiary care hospital. Perspect Clin Res. 2011;2(4):129-36.

7. Rajesh R, Vidyasagar S, Varma DM. An educational intervention to assess knowledge, Attitude, practice of pharmacovigilance among healthcare professional in an Indian tertiary care teaching hospital. Int $\mathbf{J}$ Pharm Tech Res. 2011;3(2):678-92.

8. Kaushik D. Current scenario of pharmacovigilance in India. J Drug Discovery Therapeutics. 2013;1(9):203.

9. Ganesan S, Sandhiya S, Reddy KC, Subrahmanyam DK, Adithan C. The impact of the educational intervention on knowledge, attitude, and practice of pharmacovigilance toward adverse drug reactions reporting among health-care professionals in a tertiary care hospital in South India. J Natural Sci Biol Med. 2017;8(2):203.

10. Palaian S, Ibrahim MI, Mishra P. Health professionals' knowledge, attitude and practices towards pharmacovigilance in Nepal. Pharmacy Practice. 2011;9(4):228-35.

11. Okezie EO, Olufunmilayo F. Adverse drug reactions reporting by physicians in Ibadan, Nigeria. Pharmacoepidemiol Drug Saf. 2008;17(5):517-22.

12. Gupta P, Udupa A. Adverse drug reaction reporting and pharmacovigilance: knowledge, attitudes and perceptions amongst resident doctors. J Pharmaceut Sci Res. 2011;3(2):1064.

Cite this article as: Patel NN, Patel DJ, Desai HA. Knowledge, attitude and practice of adverse drug reaction reporting among healthcare professionals of a tertiary care hospital in Gujarat. Int J Basic Clin Pharmacol 2019;8:2207-10. 